\title{
Investigation of Surgical Tool Misplacement in Virtual Fluoroscopy
}

\author{
Shinya Onogi, Toshihiro Kawase, Takaaki Sugino, and Yoshikazu Nakajima* \\ Institute of Biomaterials and Bioengineering, Tokyo Medical and Dental University, \\ 2-3-10 Kanda-Surugadai, Chiyoda, Tokyo 101-0062, Japan
}

(Received April 9, 2021; accepted June 10, 2021)

Keywords: stereoscopic navigation, virtual fluoroscopy, surgical navigation

Virtual fluoroscopy is a surgical navigation technique that is often used in orthopedic surgery. In virtual fluoroscopy, a surgeon draws tool insertion lines on intra-operative X-ray images as a surgical plan, and the position of an actual surgical tool tracked by a position sensor is also drawn on the X-ray images. The surgeon can intuitively monitor the positioning error between the plan and the tool without continuous X-ray imaging; therefore, X-ray exposure is significantly reduced compared with that in conventional fluoroscopic procedures. However, a large navigation error is often reported even when the surgeon places the tool on the planned line. To address this issue, we investigated the reason for the error by using a mock virtual fluoroscopy system. From the results of the mock operation study, we revealed that the tool positioning error depends on the convergence angle around the plan path rather than the camera rotation angle.

\section{Introduction}

Percutaneous procedures in orthopedic surgery are typical less invasive approaches for patients than traditional open techniques. On the other hand, the procedures require higher skills and greater experience from surgeons because of the lack of direct visual access of the interior of the patients during the procedures. Instead, views of the inside of patients are indirectly provided by medical images obtained by, for example, X-ray computer tomography (CT), magnetic resonance imaging (MRI), and X-ray fluoroscopy. Moreover, the increased level of X-ray exposure associated with intra-operative X-ray fluoroscopy is another concern. ${ }^{(1)}$

Surgical navigation systems, which support surgical procedures by a combination of imaging technology and computer science, can enable accurate procedures and reduce X-ray exposure. The systems generally provide virtual surgical views and information such as a surgical plan, anatomical annotation, and segmented tissues and organs. Such information is helpful for accurate and safer procedures. The clinical application of three-dimensional (3D) navigation has significantly reduced the misplacement rate of surgical tools in orthopedic surgery. ${ }^{(2)}$ However, regardless of their advantages, the navigation systems are not usually used in surgery because they require additional tasks such as 3D anatomical model generation by the segmentation of

${ }^{*}$ Corresponding author: e-mail: nakajima.bmi@tmd.ac.jp

https://doi.org/10.18494/SAM.2021.3409 
pre-operative CT images and intra-operative registration between pre-operative $\mathrm{CT}$ images and patients. Moreover, the systems require surgical invasiveness for accurate registration.

Two-dimensional (2D) navigation systems are more practical systems for use in orthopedic surgery. One of the 2D navigation systems employs computer-assisted virtual fluoroscopy ${ }^{(3-5)}$ for the placement of surgical tools such as needles, wires, and screws. Virtual fluoroscopy uses at least two fluoroscopic X-ray images and does not require images to be repeatedly taken to update instrument positions. Therefore, the exposure of the patient and the surgical team to $\mathrm{X}$-rays is reduced. Instead of multiple imaging, the instruments are superimposed on X-ray images by computation of the projected tool position from 3D positions measured by a position sensor. In contrast to 3D navigation systems, virtual fluoroscopy does not require 3D models obtained by the segmentation of pre-operative 3D volume and intra-operative registration.

Regarding the accuracy of virtual fluoroscopy, according to articles on the accuracy of pedicle screw placement by virtual fluoroscopy, pedicle breaches occurred in approximately $10-20 \%$ of cases. ${ }^{(6,7)}$ The cause of the misplacement has not been clearly explained. Addressing the misplacement is expected to lead to virtual fluoroscopy with high reliability.

The 3D measurement errors of stereoscopic systems including virtual fluoroscopy depend on the convergence angle of two stereo pairs. ${ }^{(8,9)}$ In virtual fluoroscopy, two X-ray images are taken from different angles instead of stereo pairs; therefore, error sensitivity will change with the rotation conditions of fluoroscopy. In this study, we investigated the tool placement errors by varying the convergence angle to clarify the reason for misplacements in virtual fluoroscopy.

\section{Materials and Methods}

\subsection{Virtual fluoroscopy}

Figure 1 shows a simplified configuration of virtual fluoroscopy. A position sensor with at least three trackers is used for coordinate integration among the patient, tool, and fluoroscopy. Virtual fluoroscopy depicts a virtual tool on pre-acquired X-ray images.

To provide a mathematical description of the imaging process of virtual fluoroscopy, a position sensor coordinate system $O_{G}$, a fluoroscope coordinate system $O_{\text {Fluoro }}$, a tool coordinate system $O_{T o o l}$, a patient coordinate system $O_{\text {Patient }}$, and an X-ray image pixel coordinate system $O_{\text {Xray }}$ are defined. Let $\boldsymbol{T}_{B}^{A}$ be the coordinate transformation matrix from coordinate system $B$ to coordinate system $A$, then the core process in virtual fluoroscopy is to compute the coordinate transformation among the tool and X-ray image pixel coordinate systems as follows:

$$
\boldsymbol{T}_{\text {Tool }}^{\text {Xray }}=\boldsymbol{T}_{\text {Fluoro }}^{\text {Xray }} \boldsymbol{T}_{\text {Patient }}^{\text {Fluoro }} \boldsymbol{T}_{\text {Tool }}^{\text {Patient }} \text {. }
$$

$\boldsymbol{T}_{\text {Fluoro }}^{\text {Xray }}$ is the camera projection matrix, given as

$$
\boldsymbol{T}_{\text {Fluoro }}^{\text {Xray }}=\left(\begin{array}{ccc}
f_{x} & 0 & c_{x} \\
0 & f_{y} & c_{y} \\
0 & 0 & 1
\end{array}\right),
$$




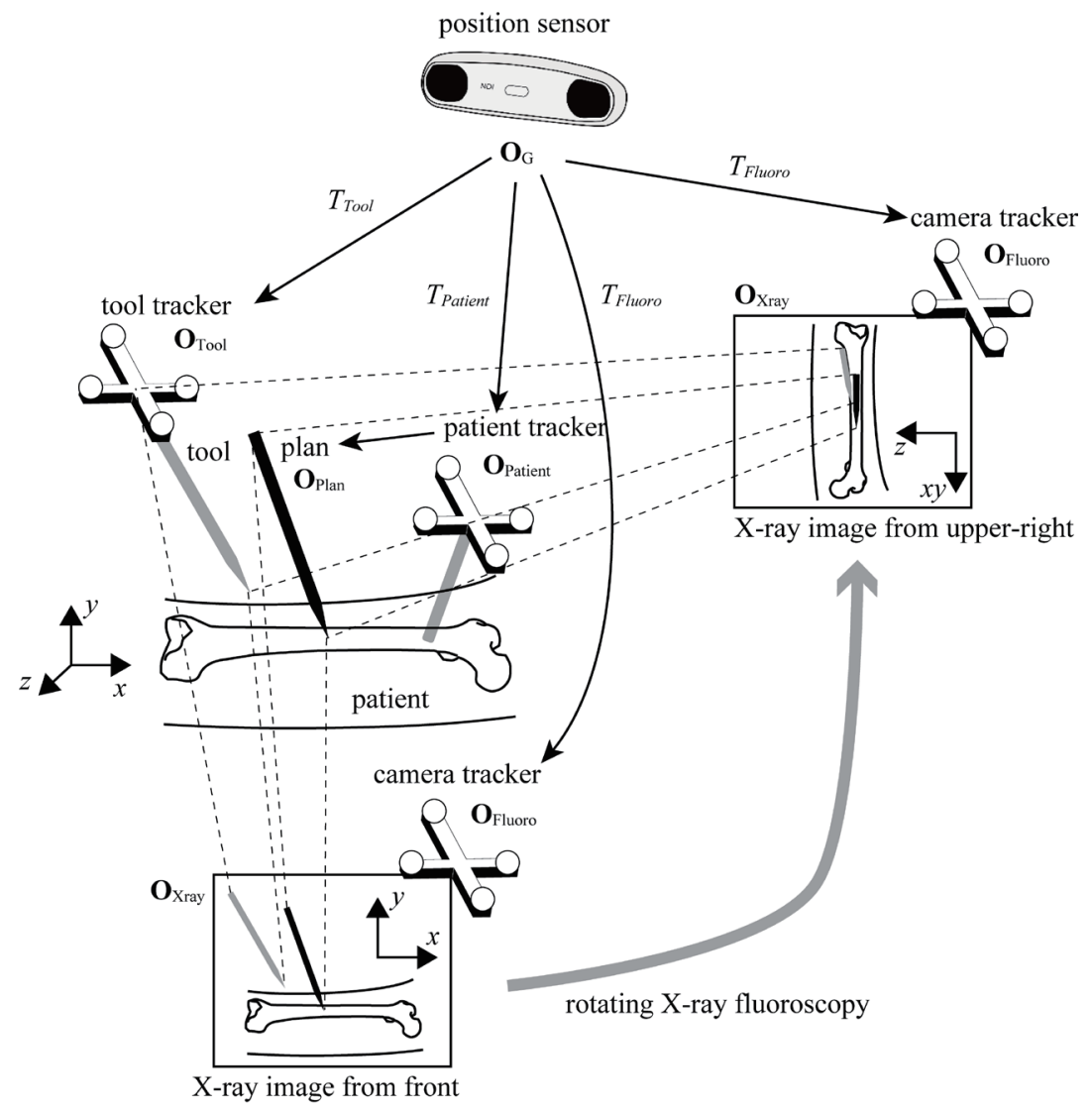

Fig. 1. Virtual fluoroscopy system.

where $f_{x}$ and $f_{y}$ are scaling factors, and $c_{x}$ and $c_{y}$ are translations from the optical axis to the upper-left corner of the image. $\boldsymbol{T}_{\text {Patient }}^{\text {Fluoro }}$ and $\boldsymbol{T}_{\text {Tool }}^{\text {Patient }}$ are transformation matrices measured by the position sensor. The camera projection matrix $\boldsymbol{T}_{\text {Fluoro }}^{\text {Xray }}$ is estimated by a camera calibration technique. ${ }^{(10)}$

We developed a mock virtual fluoroscopy system consisting of an optical position sensor (Polaris Spectra, Northern Digital Inc.), three optical trackers containing several infrared LEDs for the sensor, a USB camera with $1420 \times 720$ resolution for mock X-ray fluoroscopy, a surgical needle, a plastic femur model (Sawbones, Pacific Research Laboratories), and a Windows workstation. The optical trackers were attached to the camera, the needle, and the femur model. The navigation software was developed using Python with the well-known OpenCV library. The position sensor and the camera were connected with the workstation and controlled by the software.

\subsection{Accuracy validation protocols}

Firstly, a surgical plan $\boldsymbol{T}_{\text {Plan }}^{\text {Patient }}$ was defined for a femur model. Next, mock fluoroscopic images were acquired at $15^{\circ}$ increments of the azimuth (range: 0 to $90^{\circ}$ ) and elevation (range: 0 
to $75^{\circ}$ ) with the corresponding actual camera positions $\boldsymbol{T}_{\text {Fluoro }}^{\text {Patient }}$ measured by the position sensor. The measurements were performed twice to obtain a data set with a total of 84 images.

Error measurement tests were performed by six human subjects (not medical doctors). The experimental software randomly selected two fluoroscopic images from the data set. With the selected data, a virtual fluoroscopy view, depicting the plan and tool paths, was generated. Following the guidance of the virtual fluoroscopy, the subjects positioned the tool, then the tool position $\boldsymbol{T}_{\text {Tool }}^{\text {Patient }}$ was recorded. The tests were repeated 50 times for each subject. The placement errors $\boldsymbol{T}_{\text {Tool }}^{\text {Plan }}$ were computed as

$$
\boldsymbol{T}_{\text {Tool }}^{\text {Plan }}=\boldsymbol{T}_{\text {Patient }}^{\text {Plan }} \boldsymbol{T}_{\text {Tool }}^{\text {Patient }}
$$

The tool positioning errors were analyzed using two different convergence angles: the camera rotation angle $\theta_{\text {ca|cam }}$ and the angle around the plan path $\theta_{\text {ca|cam }}$, shown in Fig. 2. Let $O$ be the tip of the plan path, and $P_{1}$ and $P_{2}$ be the camera positions. Then, the camera convergence angle $\theta_{\text {calcam }}$ is simply given as

$$
\cos \theta_{\text {ca|cam }}=\frac{\overrightarrow{O P_{1}} \cdot \overrightarrow{O P_{2}}}{\left|\overrightarrow{O P_{1}}\right|\left|\overrightarrow{O P_{2}}\right|}
$$

On the other hand, Fig. 2(b) shows the convergence angle around the plan path. The plan coordinate system is defined with the plan path on the $z$-axis. As the camera poses, let $\alpha$ be the azimuth angles and $\beta$ be the elevation angles in the plan coordinate system. Then, $P_{1}$ and $P_{2}$ are expressed in the spherical coordinate system as

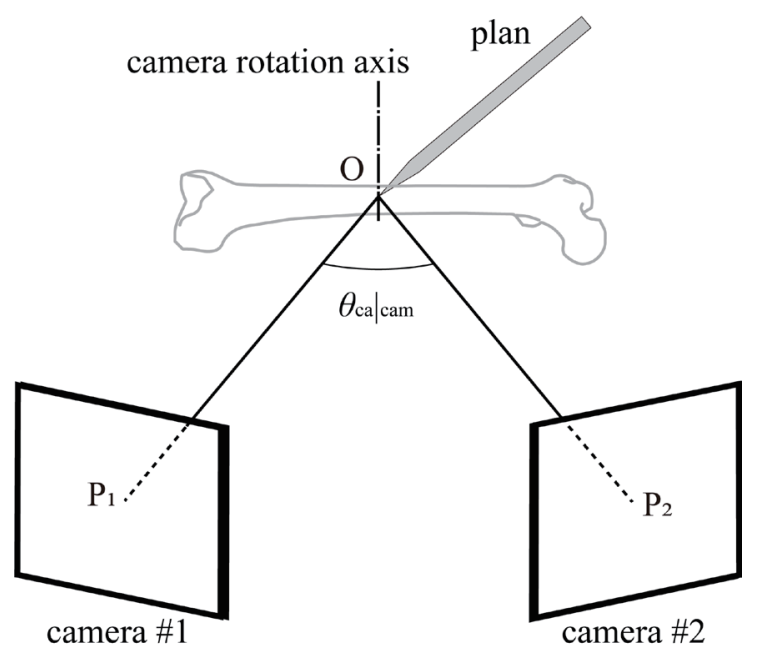

(a)

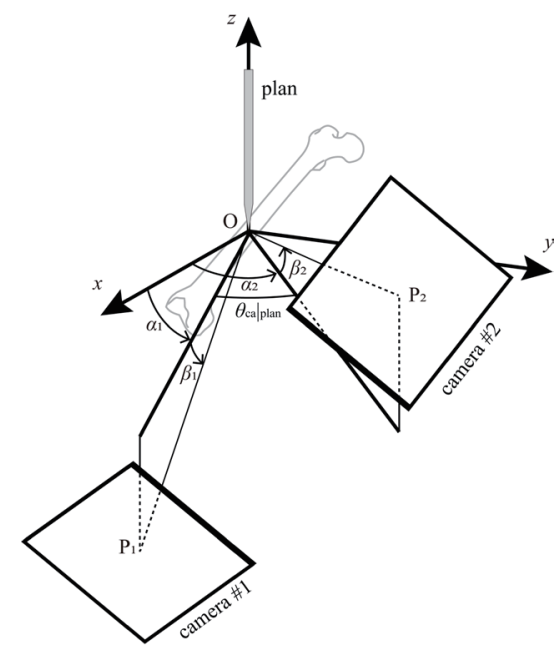

(b)

Fig. 2. Definitions of the convergence angles. (a) Camera convergence angle. (b) Convergence angle around plan path. 


$$
P(x, y, z)=r(\cos \beta \cos \alpha, \cos \beta \sin \alpha, \sin \beta),
$$

where $r$ is the distance from the origin to the camera. Using the azimuth, we determine the plan convergence angle as

$$
\cos \theta_{\text {ca|plan }}=\cos \left(\alpha_{1}-\alpha_{2}\right)=\cos \alpha_{1} \cos \alpha_{2}+\sin \alpha_{1} \sin \alpha_{2}
$$

\section{Results}

Figure 3 shows examples of navigation views before and after tool positioning, in which the black and white lines represent the plan path and the tool, respectively. Figures 4 and 5 show the obtained positioning errors as functions of the camera and plan convergence angles, respectively. The stars indicate the root-mean-square (rms) errors for each angle. Boxes show the first quartile, the third quartile, and the median, and whiskers correspond to 1.5 times the interquartile range (IQR). Figure 4 shows that there was no correlation between both the position and orientation errors and the camera angle. Meanwhile, Fig. 5 shows the errors plotted against the plan convergence angle. The rms error decreased with increasing plan convergence angle. The rms errors were well fitted by exponential functions, shown as dashed lines. Their standard

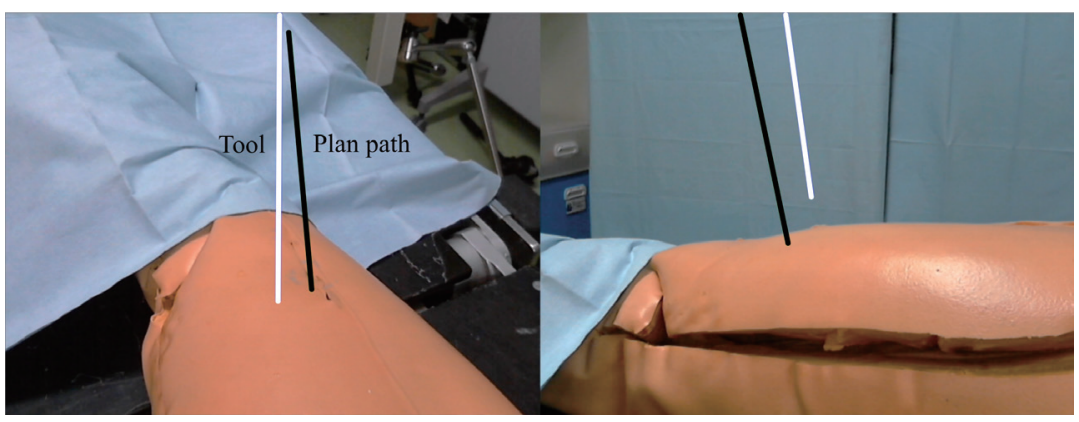

(a)

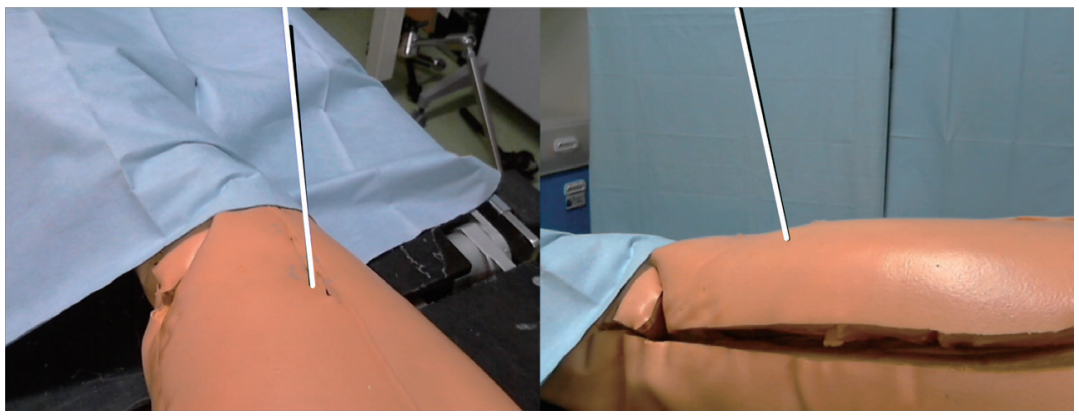

(b)

Fig. 3. (Color online) Example views of mock virtual fluoroscopy before and after tool positioning. Black and white lines represent the plan path and the tool, respectively. (a) Before tool positioning. (b) After tool positioning. 


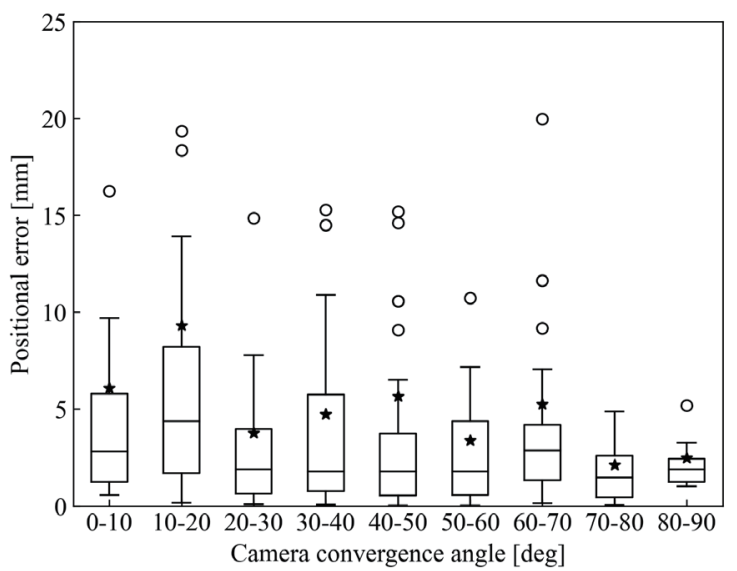

(a)

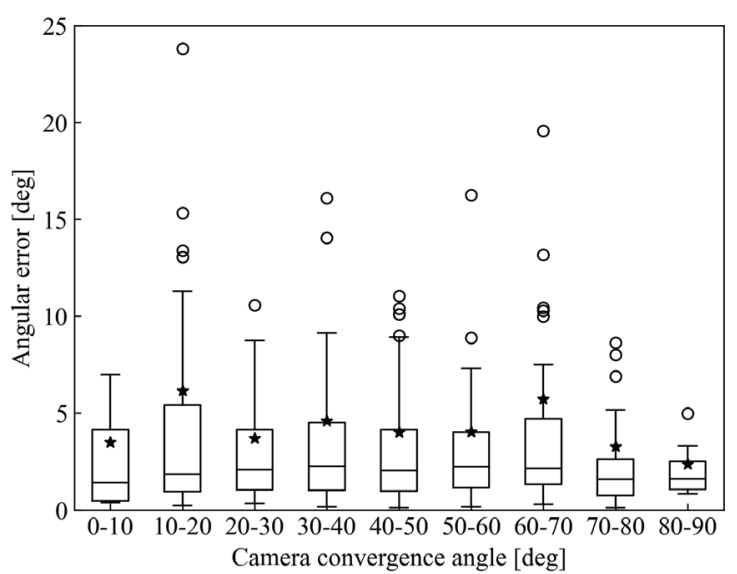

(b)

Fig. 4. Tool placement errors plotted against the camera convergence angle. Stars are rms errors.

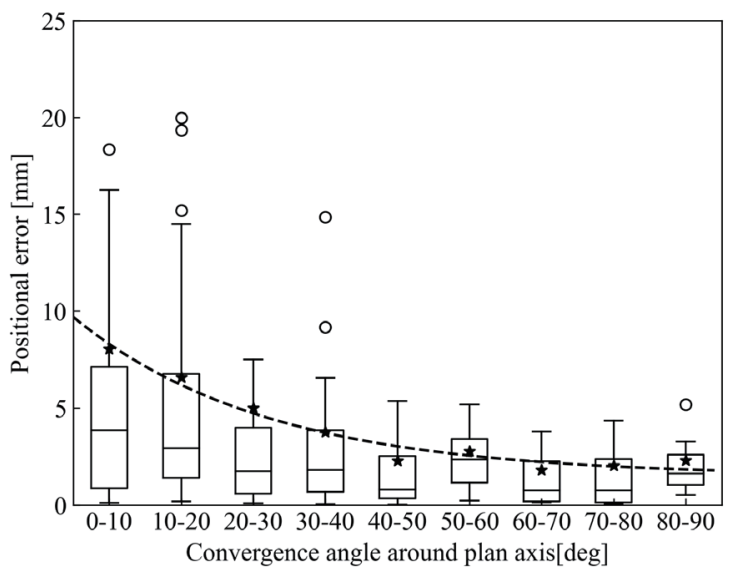

(a)

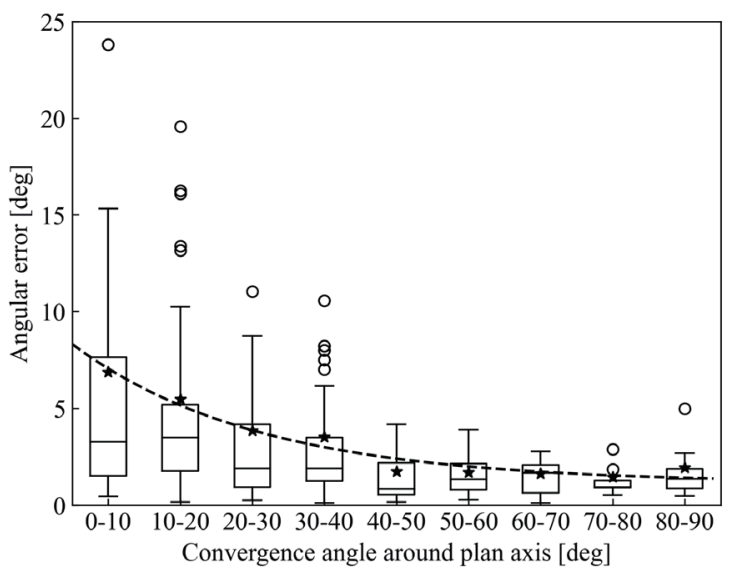

(b)

Fig. 5. Tool placement errors plotted against the convergence angle around the plan path. Stars are rms errors and dashed lines are their exponential fitted curves.

errors were $0.47 \mathrm{~mm}$ for the position and $0.46^{\circ}$ for the angle. Therefore, the misplacement of surgical tools may occur regardless of the camera convergence angle but can be reduced by increasing the convergence angle around the plan path.

\section{Discussion}

Virtual fluoroscopy visualizes 3D tool positions on 2D X-ray images. Thus, it is expected that two projection images can compensate for the depth information lost in each of the images. In this study, we revealed that the positioning error of virtual fluoroscopy is caused by the lost depth information. The results showed that the depth compensation depends on the convergence angle around a plan path rather than that around a camera rotation axis. 
Figure 6 shows the spherical coordinate system used to explain the relationship between the two types of convergence angle. In the plan coordinate system, the plan convergence angle $\theta_{\text {ca|cam }}$ is defined by the azimuth angles $\alpha_{1}$ and $\alpha_{2}$ by Eq. (6). Regarding the camera convergence angle, the substitution of Eq. (5) into Eq. (4) leads to

$$
\begin{aligned}
\cos \theta_{\text {ca|cam }} & =\left(x_{1} x_{2}+y_{1} y_{2}+z_{1} z_{2}\right) / r \\
& =\cos \beta_{1} \cos \beta_{2}\left(\cos \alpha_{1} \cos \alpha_{2}+\sin \alpha_{1} \sin \alpha_{2}\right)+\sin \beta_{1} \sin \beta_{2} .
\end{aligned}
$$

Finally, by combining Eqs. (6) and (7), the camera convergence angle is expressed in terms of the plan convergence angle as

$$
\cos \theta_{\text {ca|cam }}=\cos \beta_{1} \cos \beta_{2} \cos \theta_{\text {ca|plan }}+\sin \beta_{1} \sin \beta_{2} .
$$

Therefore, the elevation is the factor causing the angular difference between the two types of convergence angle. In other words, the elevation angle does not contribute to the accuracy. Figure 7 shows a comparison of the two types of convergence angle in the experiment. It indicates that the plan convergence angle is not sufficiently large even for a large camera rotation angle, which is due to the elevation.

The results explain why surgical tool misplacements such as pedicle breaches are more likely to occur for a small convergence angle around the plan path, even for sufficient rotation in $\mathrm{C}$-arm fluoroscopy. Figure 8 shows typical fluoroscope motion during anatomical and navigation processes. To monitor the tool insertion condition in surgery, a fluoroscope is rotated around a femur as shown in Fig. 8(a). To achieve the motion conveniently, C-arm fluoroscopy has a rotation mechanism around the horizontal axis. Therefore, tool misplacement seems to randomly occur in virtual fluoroscopy even when fluoroscopic images are taken with a sufficient rotation

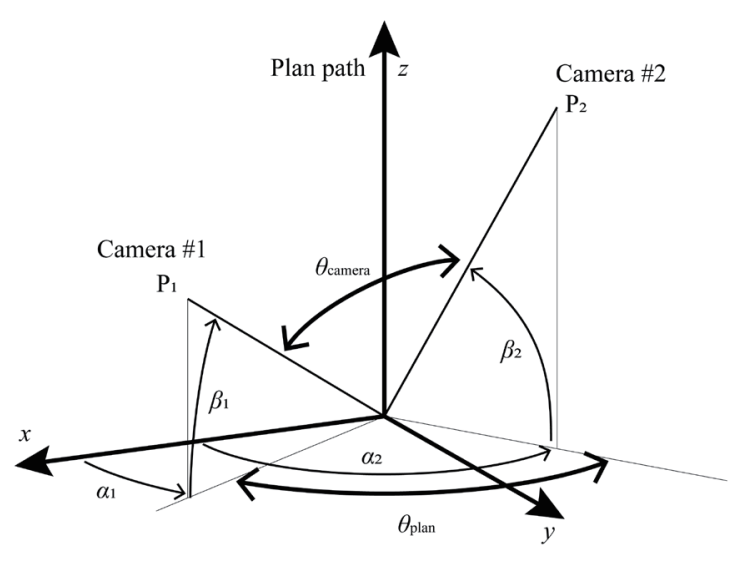

Fig. 6. Two types of convergence angle expressed in spherical coordinate system.

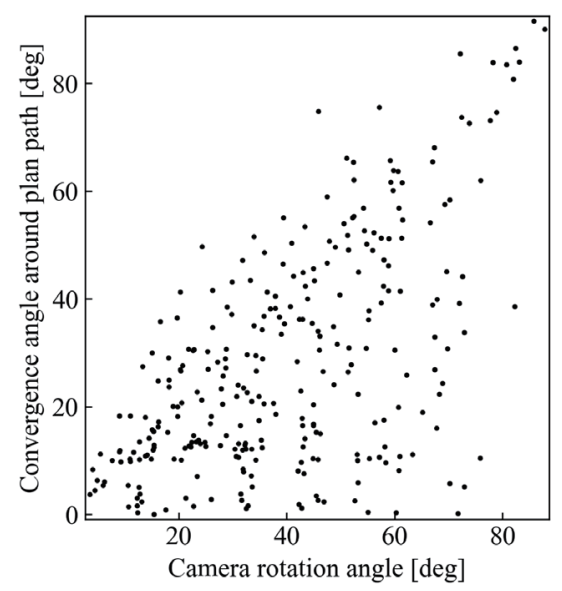

Fig. 7. Comparison between camera rotation angles and angles around plan paths. 


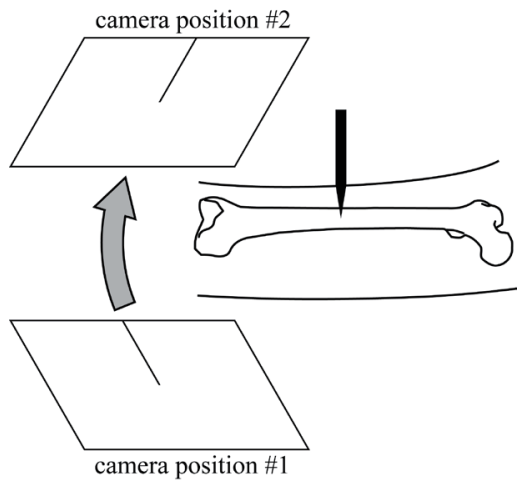

(a)

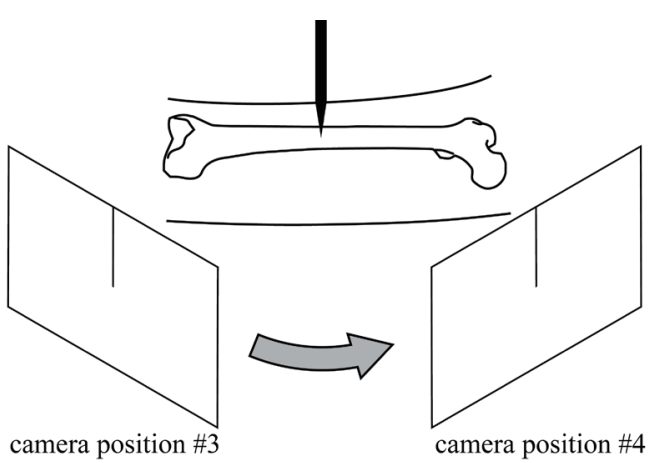

(b)

Fig. 8. Two types of camera rotation motion. (a) Rotation around anatomical axis. (b) Rotation around plan path.

angle. In studies that evaluated the accuracy of virtual fluoroscopy in clinical settings, ${ }^{(2,3)} \mathrm{X}$-ray images at the lateral and anteroposterior planes were used for virtual fluoroscopy as shown in Fig. 8(a). To obtain a sufficient convergence angle around the plan path, the fluoroscope should be rotated around the plan path, as shown in Fig. 8(b).

In this study, we used a general USB camera instead of an X-ray fluoroscope, and the camera projection parameters will be different for the two devices. However, an X-ray fluoroscope causes less distortion than an optical camera; therefore, our experiments do not underestimate the errors.

The experiments were performed by untrained human subjects. It is possible that well-trained doctors will perform the tool positioning more accurately. Note that because the tool plans were randomly selected, the results did not include learning effects.

Potential navigation errors due to the imaging conditions should be estimated to ensure reliable procedures. Thus, a theoretical error estimation is needed rather than an estimation by an empirical relationship. Therefore, our future work will focus on modeling the potential navigation error in virtual fluoroscopy toward achieving highly accurate and reliable surgery.

\section{Conclusions}

We investigated the navigation errors of virtual fluoroscopy by comparing camera and plan convergence angles. The results suggested that a small convergence angle around the plan path resulted in a large placement error regardless of the camera rotation angle. We revealed that the plan convergence angle is important for reducing misplacement in virtual fluoroscopy.

\section{References}

1 R. Sanders, K. J. Koval, T. DiPasquale, G. Schmelling, S. Stenzler, and E. Ross: J. Bone Joint Surg. Am. 75 (1993) 326. https://doi.org/10.2106/00004623-199303000-00003K

2 T. Laine, T. Lund, M. Ylikoski, J. Lohikoski, and D. Schlenzka: Eur. Spine J. 9 (2000) 235. https://doi. org/10.1007/s005860000146 
3 R. Hofstetter, M. Slomczykowski, M. Sati, and L. P. Nolte: Comput. Aided Surg. 4 (1999) 65. https://doi. org/10.1002/(SICI)1097-0150(1999)4:2<65::AID-IGS1>3.0.CO;2-Y

4 N. Suhm, A. L. Jacob, L. P. Nolte, P. Regazzoni, and P. Messmer: Comput. Aided Surg. 5 (2000) 391. https://doi. org/10.1002/igs.1001

5 Y. R. Rampersaud, J. H. Pik, D. Salonen, and S. Farooq: Spine 30 (2005) E183. https://doi.org/10.1097/01. brs.0000157490.65706.38

6 S. Rajasekaran, S. Vidyadhara, P. Ramesh, and A. P. Shetty: Spine 32 (2007) E56. https://doi.org/10.1097/01. brs.0000252094.64857.ab

7 B. Ravi, A. Zahrai, and R. Rampersaud: Spine 36 (2011) 84. https://doi.org/10.1097/BRS.0b013e3181cbfd09

8 D. Weinshall: Comput. Vision Graphics Image Process. 49 (1990) 222. https://doi.org/10.1016/0734189X(90)90138-L

9 S. D. Blostein and T. S. Huang: IEEE Trans. Pattern Anal. Mach. Intell. 9 (1987) 752. https://doi.org/10.1109/ TPAMI.1987.4767982

10 Z. Zhang: IEEE Trans. Pattern Anal. Mach. Intell. 22 (2000) 1330. https://doi.org/10.1109/34.888718

\section{About the Authors}

Shinya Onogi received his B.S. and M.S. degrees from Hokkaido University, Japan, in 2002 and 2004, respectively, and his Ph.D. degree from the University of Tokyo, Japan, in 2007. From 2007 to 2010, he was a project researcher at the University of Tokyo. From 2010 to 2011, he was a postdoctoral fellow at Johns Hopkins University, USA. From 2011 to 2014, he was an assistant professor at Tokyo University of Agriculture and Technology. From 2014 to 2018, he was an associate professor at Kyushu University, Japan. From 2018 to 2020, he was an associate professor at Fukuoka Institute of Technology, Japan. Since 2020, he has been an associate professor at Tokyo Medical and Dental University. His research interests are in biomedical engineering, mechatronics, and system engineering. (shinya.onogi.1979@ieee.org)

Toshihiro Kawase received his B.S., M.S., and Ph.D. degrees from Tokyo Institute of Technology, Tokyo, Japan, in 2007, 2009, and 2012, respectively. He was a research fellow with the Research Institute of National Rehabilitation Center for Persons with Disabilities from 2012 to 2015, and worked as a postdoctoral fellow and a specially appointed assistant professor with Tokyo Institute of Technology from 2015 to 2017. He is currently an assistant professor with Tokyo Medical and Dental University and Tokyo Institute of Technology. His research interests include medical robots, rehabilitation robotics, and biological signal processing.

(kawase.bmi@tmd.ac.jp)

Takaaki Sugino received his B.S., M.S., and Ph.D. degrees from Chiba University, Japan, in 2012, 2014, and 2017, respectively. From 2017 to 2019, he worked as a project researcher at Nagoya University, Japan. Since 2019, he has been an assistant professor at Tokyo Medical and Dental University, Japan. His research interests are in medical image analysis and computeraided diagnosis and surgery. (sugino.bmi@tmd.ac.jp) 
Yoshikazu Nakajima received his Ph.D. degree from Osaka University, Japan, in 1997. He worked for the head office and Information Technology R\&D Center of Mitsubishi Electric Corporation from 1997 to 2000, then for the Division of Functional Diagnostic Imaging, Graduate School of Medicine, Osaka University, as an assistant professor from 2001 to 2005. He then worked as an associate professor at the Department of Bioengineering and Intelligent Modeling Laboratory of the University of Tokyo from 2005 to 2017. Currently, he is a professor at the Institute of Biomaterials and Bioengineering, Tokyo Medical and Dental University. (nakajima.bmi@tmd.ac.jp) 